\title{
On the non-emptiness of the fuzzy core
}

\author{
Citation for published version (APA):
}

Allouch, N., \& Predtetchinski, A. (2005). On the non-emptiness of the fuzzy core. METEOR, Maastricht University School of Business and Economics. METEOR Research Memorandum No. 003

https://doi.org/10.26481/umamet.2005003

Document status and date:

Published: 01/01/2005

DOI:

10.26481/umamet.2005003

Document Version:

Publisher's PDF, also known as Version of record

\section{Please check the document version of this publication:}

- A submitted manuscript is the version of the article upon submission and before peer-review. There can be important differences between the submitted version and the official published version of record.

People interested in the research are advised to contact the author for the final version of the publication, or visit the DOI to the publisher's website.

- The final author version and the galley proof are versions of the publication after peer review.

- The final published version features the final layout of the paper including the volume, issue and page numbers.

Link to publication

\footnotetext{
General rights rights.

- You may freely distribute the URL identifying the publication in the public portal. please follow below link for the End User Agreement:

www.umlib.nl/taverne-license

Take down policy

If you believe that this document breaches copyright please contact us at:

repository@maastrichtuniversity.nl

providing details and we will investigate your claim.
}

Copyright and moral rights for the publications made accessible in the public portal are retained by the authors and/or other copyright owners and it is a condition of accessing publications that users recognise and abide by the legal requirements associated with these

- Users may download and print one copy of any publication from the public portal for the purpose of private study or research.

- You may not further distribute the material or use it for any profit-making activity or commercial gain

If the publication is distributed under the terms of Article $25 \mathrm{fa}$ of the Dutch Copyright Act, indicated by the "Taverne" license above, 


\title{
On the non-emptiness of the fuzzy core *
}

\author{
Nizar Allouch \\ Department of Economics \\ Queen Mary, University of London \\ Mile End Rd, E1 4NS \\ London, United Kingdom \\ n.allouch@qmul.ac.uk
}

\author{
Arkadi Predtetchinski \\ Department of Economics \\ Maastricht University \\ P.O. Box 616, 6200 MD \\ Maastricht, the Netherlands \\ a.predtetchinski@algec.unimaas.nl
}

Current Version: January, 2005

\begin{abstract}
The seminal contribution of Debreu-Scarf [4] connects the two concepts of core and competitive equilibrium in exchange economies. In effect, their core-equilibrium equivalence result states that, when the set of economic agents is replicated, the set of core allocations of the replica economy shrinks to the set of competitive allocations. Florenzano [6] defines the fuzzy core as the set of allocations which cannot be blocked by any coalition with an arbitrary rate of participation and then shows the asymptotic limit of cores of replica economies coincides with the fuzzy core. In this note, we provide an elementary proof of the non-emptiness of the fuzzy core for an exchange economy. Unlike the classical Debreu-Scarf limit theorem [4] and its numerous extensions our result does not require any asymptotic intersection -or limit- of the set of core allocations of replica economies.
\end{abstract}

JEL classification codes: D51, C71.

Keywords: fuzzy core, payoff-dependent balancedness, exchange economies.

${ }^{*}$ Helpful comments of Jean-Jacques Herings, Hans Peters and Myrna Wooders are gratefully acknowledged. 


\section{Introduction}

The core is a meaningful solution concept for market economies. If an allocation of resources has the property that a group of people is able to achieve a preferred outcome for its members, using only the resources of the group membership, then it is reasonable to assume that the group will attempt to block that allocation. In this sense, allocations not in the core are unstable. While competitive price-taking equilibrium outcomes are in the core, in general the core is larger than the set of equilibrium allocations. The question of the relationship of the core of an economy to the set of competitive equilibrium allocations originates with Edgeworth's [5] conjecture that, if an economy were replicated, the core of an economy would shrink to the set of competitive equilibrium allocations. The seminal contribution of Debreu-Scarf [4] gives a rigorous treatment of Edgeworth's conjecture [5]. The underlying idea is that, if the number of agents in the market is sufficiently large, no single agent can have monopoly power. Consequently, a competitive price-taking equilibrium will be sustained.

Florenzano [6], following Aubin's [2] notion of fuzzy coalitions, defines the notion of a fuzzy core as the set of allocations that cannot be blocked by any coalition with an arbitrary rate of participation. Under a weak continuity assumption on preference relations, the asymptotic limit of core of replica economics coincides with the fuzzy core. Accordingly, in the context of a private-ownership production economy with nontransitive preference relations, Florenzano [6] establishes the nonemptiness of the fuzzy core. By enlarging the feasible payoff sets for coalitions, Predtetchinski [8] provides an alternative proof of the non-emptiness of the fuzzy core. Notwithstanding the novelty of this approach, the result still relies on a conventional limit argument.

In this note our main contribution is to provide an elementary proof of non-emptiness of the fuzzy core. In particular, unlike the Debreu- Scarf result and its numerous extensions (see Aliprantis etal [1], Florenzano [6], and Predtetchinski [8] amongst others) our result does not require any asymptotic intersection. Instead of allowing the economy to become large through replication, we enlarge the set of feasible payoffs for the economy in utility space. We define feasible payoffs as those achievable by coalitions with arbitrary compositions of their memberships, where the rate of participation of an individual player in a coalition is expressed as a proportion. This setting is closely related to the one introduced by Predtetchinski [8], with two exceptions. First, our setting does not require a lower bound on each rate of participation in fuzzy coalitions. Second, rather than using recent results on the payoff-dependent balancedness and the core due to Bonnisseau-Iehle [3] and Predtetchinski-Herings [7], our result is established directly by using the Fan coincidence theorem. In the sense that our proof uses elementary arguments, it makes the same sort of contribution for the fuzzy core as that of Vohra [10] and Shapley and Vohra [9] for the core. A novel feature of our proof is that in our fixed point argument it is essential to trace some payoffs back to their corresponding participation rates.

The note is organized as follows. Section 2 summarizes the notation. Section 3 is devoted to the basic model and definitions and Section 4 is dedicated to the proof of the result. We will show that the fuzzy core of an exchange economy is non-empty. 


\section{Notation}

Let $n$ be a positive integer and $N$ be the set of integers $\{1, \ldots, n\}$. The symbol $\mathbb{R}^{N}$ denotes the space of functions $x: i \in N \mapsto x^{i} \in \mathbb{R}$. Let $\Delta$ denote the unit simplex in $\mathbb{R}^{N}$, thus $\Delta=\left\{\pi \in \mathbb{R}^{N} \mid \pi^{i} \geq 0\right.$ for all $i \in N$ and $\left.\sum_{i \in N} \pi^{i}=1\right\}$. For each point $\pi$ in $\Delta$ let $\operatorname{supp}(\pi)$ denote the set $\left\{i \in N \mid \pi_{i}>0\right\}$. Let $\beta$ denote the barycenter of $\Delta$. For a subset $A$ of $\mathbb{R}^{N}$, the symbols int $A$ and $\partial A$ denote, respectively, the interior and the boundary of $A$.

\section{Fuzzy core}

We consider a pure exchange economy $E$ in which the set of agents is $N$, and the commodity space is $\mathbb{R}^{l}$. Each agent $i \in N$ is characterized by a consumption set $X^{i} \subseteq \mathbb{R}^{l}$, initial endowment of commodities $e^{i} \in \mathbb{R}^{l}$, and a utility function $u^{i}: X^{i} \rightarrow \mathbb{R}$. We employ the following assumptions:

(A1) For each $i \in N$, the set $X^{i}$ is a non-empty closed convex and bounded below subset of $\mathbb{R}^{l}$ containing $e^{i}$.

(A2) For each $i \in N$ the utility function $u^{i}$ is a continuous and quasi-concave function.

Let us denote the product $\times_{i \in N} X^{i}$ by $X$. Let $F(E)$ denote the set $\left\{x \in X \mid \sum_{i \in N} x^{i} \leq\right.$ $\left.\sum_{i \in N} e^{i}\right\}$ of commodity allocations that are feasible for the grand coalition $N$.

Definition 1 An allocation $x \in F(E)$ is an element of the fuzzy core $C^{f}(E)$ of the economy $E$ if there exist no $\pi \in \Delta$ and no $\chi \in X$ such that $\sum_{i \in N} \pi^{i} \chi^{i} \leq \sum_{i \in N} \pi^{i} e^{i}$ and $u^{i}\left(x^{i}\right)<$ $u^{i}\left(\chi^{i}\right)$ for all $i \in \operatorname{supp}(\pi)$.

The main result of this paper is the following theorem.

Theorem 1 The fuzzy core of the economy $E$ is non-empty.

In the next section we establish a characterization of the fuzzy core in the utility space and prove Theorem 1. Our construction resembles that in Predtetchinski [8], but is different in two ways. First, we remove an exogenous lower bound on the participation rates in a fuzzy coalition, thus obtaining a direct characterization of the fuzzy core that does not rely on any limit argument. Second, our proof is independent of the non-emptiness result for the core with additional requirements in Bonnisseau and Iehle [3] and is based ultimately on the Fan's coincidence theorem. 


\section{The non-emptiness of the fuzzy core}

Define the subset $V$ of $\mathbb{R}^{N}$ by the equation

$$
V=\left\{v \in \mathbb{R}^{N} \mid \begin{array}{c}
\text { There exist } x \in X \text { and } \pi \in \Delta \text { such that } \\
\sum_{i \in N} \pi^{i} x^{i} \leq \sum_{i \in N} \pi^{i} e^{i} \\
v^{i} \leq u^{i}\left(x^{i}\right) \text { for all } i \in \operatorname{supp}(\pi)
\end{array}\right\} .
$$

Proposition 1 The set $V$ is a non-empty closed and proper subset of $\mathbb{R}^{N}$. Moreover, it is comprehensive from below: if $\bar{v} \in V, v \in \mathbb{R}^{N}$ and $v^{i} \leq \bar{v}^{i}$ for all $i \in N$, then $v \in V$.

Proof. The non-emptiness, the properness and comprehensiveness from below of the set $V$ are immediate from the definition. We establish the closedness of $V$.

For each player $i$, choose a vector $c^{i}$ in $\mathbb{R}^{l}$ such that $c^{i} \leq x^{i}$ for all $x^{i} \in X^{i}$. Let $v_{q}$ be a sequence of points in $V$ converging to a point $v$ of $\mathbb{R}^{N}$. We must show that $v$ is an element of $V$. For each $q$, let $x_{q} \in X$ be a commodity allocation and $\pi_{q} \in \Delta$ be a fuzzy coalition such that

$$
\begin{array}{r}
\sum_{i \in N} \pi_{q}^{i} x_{q}^{i} \leq \sum_{i \in N} \pi_{q}^{i} e^{i} \\
v_{q}^{i} \leq u^{i}\left(x_{q}^{i}\right) \text { for all } i \in \operatorname{supp}\left(\pi_{q}\right) .
\end{array}
$$

Replacing if necessary the sequences by subsequences, suppose that $\pi_{q}$ converges to a point $\pi$ of $\Delta$. Now we argue that for each $j \in \operatorname{supp}(\pi)$ the sequence $x_{q}^{j}$ is bounded from above as well as from below. Indeed, for all $q$ large enough we have the chain of inequalities

$$
\frac{\pi^{j}}{2}\left[x_{q}^{j}-c^{j}\right] \leq \pi_{q}^{j}\left[x_{q}^{j}-c^{j}\right] \leq \sum_{i \in N} \pi_{q}^{i}\left[x_{q}^{i}-c^{i}\right] \leq \sum_{i \in N} \pi_{q}^{i}\left[e^{i}-c^{i}\right] \leq \sum_{i \in N}\left[e^{i}-c^{i}\right],
$$

establishing the existence of an upper bound for the sequence $x_{q}^{j}$. Once again, replacing the sequences by subsequences, we can assume that the sequence $x_{q}^{i}$ converges to a point $x^{i}$ of $X^{i}$ for all $i \in \operatorname{supp}(\pi)$.

Inequality (1) implies the inequality

$$
\sum_{i \in N \backslash \operatorname{supp}(\pi)} \pi_{q}^{i} c^{i}+\sum_{i \in \operatorname{supp}(\pi)} \pi_{q}^{i} x_{q}^{i} \leq \sum_{i \in N} \pi_{q}^{i} e^{i}
$$

Taking the limits in Inequalities (2) and (3) yields

$$
\begin{gathered}
\sum_{i \in \operatorname{supp}(\pi)} \pi^{i} x^{i} \leq \sum_{i \in \operatorname{supp}(\pi)} \pi^{i} e^{i} \\
v^{i} \leq u^{i}\left(x^{i}\right) \text { for all } i \in \operatorname{supp}(\pi) .
\end{gathered}
$$

This implies that $v$ is element of $V$, as desired. 
Define the correspondence $\Pi: V \rightrightarrows \Delta$ by the equation

$$
\Pi(v)=\left\{\begin{array}{l|l}
\pi \in \Delta & \begin{array}{c}
\text { There exist } x \in X \text { such that } \\
\sum_{i \in N} \pi^{i} x^{i} \leq \sum_{i \in N} \pi^{i} e^{i} \\
v^{i} \leq u^{i}\left(x^{i}\right) \text { for all } i \in \operatorname{supp}(\pi)
\end{array}
\end{array}\right\} .
$$

Proposition 2 For each $v \in V$ the set $\Pi(v)$ is non-empty and convex. The correspondence $\Pi$ has a closed graph.

The proof of this proposition is similar to that of Proposition 3 in Predtetchinski [8].

The following proposition summarizes the relationship between the fuzzy core on the one hand, and the set $V$ and the correspondence $\Pi$, on the other hand. Its proof is straightforward and is therefore omitted.

Proposition 3 Let $x$ be an allocation in the fuzzy core of the economy $E$ and let $v \in \mathbb{R}^{N}$ be given by $u^{i}\left(x^{i}\right)=v^{i}$ for all $i \in N$. Then $v \in \partial V$ and $\beta \in \Pi(v)$. Conversely, let $v \in \partial V$ be such that $\beta \in \Pi(v)$. Then there is an allocation $x$ in the fuzzy core of the economy $E$ such that $v^{i} \leq u^{i}\left(x^{i}\right)$ for all $i \in N$.

Under the maintained assumptions $F(E)$ is a compact set. Therefore, for each $i \in N$ there is a number $\bar{m}^{i}$ such that $u^{i}\left(x^{i}\right)<\bar{m}^{i}$ for all $x \in F(E)$. For each $i \in N$ also choose a number $\underline{m}^{i}$ such that $\underline{m}^{i}<u^{i}\left(e^{i}\right)$. Define $\bar{V}$ denote the set

$$
\bar{V}=V \cap\left\{i \in \mathbb{R}^{N} \mid v^{i} \leq \bar{m}^{i} \text { for all } i \in N\right\} .
$$

A point $v \in V$ belongs to the boundary of $\bar{V}$ if and only if either $v \in \partial V$ or $v^{i}=\bar{m}^{i}$ for some $i \in N$, see Figure 1 .

Proposition 4 There is a homeomorphism $h$ from the space $\Delta$ to the space $\partial \bar{V} \cap\{v \in$ $\mathbb{R}^{N} \mid v^{i} \geq \underline{m}^{i}$ for all $\left.i \in N\right\}$ such that $h^{i}(\pi)=\underline{m}^{i}$ whenever $\pi \in \Delta$ and $i \in N-\operatorname{supp}(\pi)$.

Proof. Let $\pi \in \Delta$ be given. Let $R$ be the ray emanating from the point $\underline{m}=\left(\underline{m}^{1}, \ldots \underline{m}^{n}\right)$ in the direction of $\pi$. Thus, every point $r$ of $R$ is of the form $r=\underline{m}+t \pi$ for some nonnegative real number $t$. First we argue that the ray $R$ intersects the boundary of $\bar{V}$ at exactly one point.

To see that $R$ does intersect $\partial \bar{V}$, observe that the point $\underline{m}$ belongs to both to the set $\bar{V}$ and to the ray $R$. Thus, the set $R_{1}=R \cap \bar{V}$ is non-empty. Furthermore, for any $i \in \operatorname{supp}(\pi)$, there is an $r \in R$ such that $r^{i} \geq \bar{m}^{i}$, so that $r$ lies outside the interior of $\bar{V}$. Therefore, the set $R_{2}=R \cap\left(\mathbb{R}^{N}-i n t \bar{V}\right)$ is non-empty. Thus $R_{1}$ and $R_{2}$ are the non-empty closed subsets of $R$ whose union is $R$. By connectedness of $R$, the set $R_{1} \cap R_{2}=R \cap \partial \bar{V}$ must be non-empty.

Suppose that the set $R \cap \partial \bar{V}$ contains two distinct points $\underline{v}$ and $\bar{v}$. Thus $\underline{v}=\underline{m}+\underline{t} \pi$ and $\bar{v}=\underline{m}+\bar{t} \pi$ for some non-negative reals $\underline{t}$ and $\bar{t}$. Without a loss of generality we can 
assume that $\underline{t}<\bar{t}$. For each $i \in N-\operatorname{supp}(\pi)$, we then have $\underline{v}^{i}=\underline{m}^{i}<\bar{m}^{i}$. For each $i \in \operatorname{supp}(\pi)$, we have $\underline{v}^{i}<\bar{v}^{i} \leq \bar{m}^{i}$. The set

$$
\bigcup_{i \in N}\left\{v \in \mathbb{R}^{N} \mid v^{i} \leq u^{i}\left(e^{i}\right)\right\}
$$

is entirely contained in $V$. Therefore, if $v$ is a point of $\mathbb{R}^{N}$ with some coordinate $v^{i}$ equal to $\underline{m}^{i}$, it is contained in the interior of $V$. It follows that if $N-\operatorname{supp}(\pi)$ is non-empty, then the point $\underline{v}$ is contained in the interior of $V$. If $\operatorname{supp}(\pi)=N$, then because $\underline{v}^{i}<\bar{v}^{i}$ for all $i \in N$ and $\bar{v} \in V$, we know that $\underline{v}$ lies in the interior of $V$. We have thus demonstrated that the inequality $\underline{v}^{i}<\bar{m}^{i}$ holds for all $i \in N$ and that $\underline{v}$ is in the interior of $V$. It follows that $\underline{v}$ is in the interior of $\bar{V}$, a contradiction.

Define the map $h$ by letting $h(\pi)$ be the unique point in the intersection of the ray $R$ and the set $\partial \bar{V}$. We now demonstrate that $h$ has an inverse. Let $g$ denote a map from $\partial \bar{V} \cap\left\{v \in \mathbb{R}^{N} \mid v^{i} \geq \underline{m}^{i}\right.$ for all $\left.i \in N\right\}$ to $\Delta$ given by the equation

$$
g(v)=\frac{v-\underline{m}}{\sum_{i \in N}\left(v^{i}-\underline{m}^{i}\right)} .
$$

The denominator in the right-hand side is always positive, because the point $\underline{m}$ lies in the interior of $\bar{V}$. It is easy to see that $g$ is indeed the inverse of $h$, that is $h \circ g$ and $g \circ h$ are equal to the respective identity maps.

Clearly, $g$ is a continuous map. Furthermore, because its domain is compact and the codomain is Hausdorff, it carries closed sets to closed sets. Therefore, also $h$ is a continuous map. This proves that $h$ is a homeomorphism.

The rest of the proof relies on a version of the Fan's coincidence theorem, as stated below. Given a non-empty and convex subset $Y$ of $\mathbb{R}^{N}$ and a point $y$ of $Y$, let $N(Y, y)=\{z \in$ $\mathbb{R}^{N} \mid\left(y-y^{\prime}\right)^{\top} z \geq 0$ for all $\left.y^{\prime} \in Y\right\}$ denote the normal cone of the set $Y$ at the point $y$. A zero point of a correspondence $\Phi: Y \rightrightarrows \mathbb{R}^{N}$ is a point $y$ of $Y$ such that $\Phi(y)$ contains the zero.

Theorem 2 Let $Y$ be a non-empty compact and convex subset of $\mathbb{R}^{N}$. Let $\Phi: Y \rightrightarrows \mathbb{R}^{N}$ be a correspondence with non-empty convex values having a compact graph. Suppose that for every $y \in Y$ and for every $z \in N(Y, y)$ there exists $a \phi \in \Phi(y)$ such that $z^{\top} \phi \leq 0$. Then $\Phi$ has a zero point.

Proposition 5 There exists $v^{*} \in \partial V$ such that $\beta \in \Pi\left(v^{*}\right)$.

Proof. It is sufficient to prove that there is a point $v^{*} \in \partial \bar{V}$ such that $\beta \in \Pi\left(v^{*}\right)$. For this would mean that there is a point $x \in F(E)$ such that $v^{i} \leq u^{i}\left(x^{i}\right)$ for all $i \in N$, implying that $v^{i}<\bar{m}^{i}$ for all $i \in N$. Therefore, $v^{*} \in \partial V$, as desired.

Let $h$ be a homeomorphism of Proposition 4. Define the correspondence $\Phi: \Delta \rightrightarrows \mathbb{R}^{N}$ by letting $\Phi(\pi)=\Pi(h(\pi))-\{\beta\}$ for all $\pi \in \Delta$. Clearly, the correspondence $\Phi$ has nonempty and convex values. Its graph is closed, because $h$ is continuous and the graph of $\Pi$ 
is closed. Since $\Phi$ maps a compact set $\Delta$ into a compact set $\Delta-\{\beta\}$, its graph is, in fact, a compact set.

We now verify the boundary condition of the Fan's coincidence theorem. Let $\pi \in \Delta$ be given. Let $v$ denote the vector $h(\pi)$ and let $S$ denote the (possibly empty) set $N-\operatorname{supp}(\pi)$. Then the normal cone of $\Delta$ at $\pi$ is given by the set

$$
N(\Delta, \pi)=\left\{z \in \mathbb{R}^{n} \mid z=a \mathbf{1}+\sum_{i \in S} l_{i} \mathbf{r}_{i}, a \in \mathbb{R}, l_{i} \leq 0\right\},
$$

where $\mathbf{1}=(1, \ldots, 1)$ and $\left(\mathbf{r}_{1}, \ldots, \mathbf{r}_{n}\right)$ is the standard ordered basis of $\mathbb{R}^{N}$. Let $z \in N(\Delta, \pi)$ be given. If $\pi$ belongs to the relative interior of $\Delta$ so that $\operatorname{supp}(\pi)=N$, then $z$ is proportional to the vector 1 . In this case the inequality $z^{\top} \phi=0$ holds for every $\phi \in \Phi(\pi)$.

Consider now the case where $\pi$ lies on the relative boundary of $\Delta$, so that the set $S$ is non-empty. Then $v^{i}=\underline{m}^{i}$ and therefore $v^{i}<u^{i}\left(e^{i}\right)$ for all $i \in S$. But this implies that the set $\Pi(v)$ contains the entire face $\Delta_{S}=\left\{\bar{\pi} \in \Delta \mid \bar{\pi}^{i}=0\right.$ for all $\left.i \in N \backslash S\right\}$ of the simplex $\Delta$. In particular, $\Pi(v)$ contains the barycenter $\beta_{S}$ of $\Delta_{S}$. The vector $\phi=\beta_{S}-\beta$ is therefore an element of $\Phi(\pi)$. Since $0 \leq \phi^{i}$ for all $i \in S$, the inequality $\phi^{\top} z \leq 0$ holds.

By the Fan's coincidence theorem, the correspondence $\Phi$ has a zero point, say $\pi^{*}$. Letting $v^{*}$ be equal to $h\left(\pi^{*}\right)$, we see that $v^{*} \in \partial \bar{V}$ and $\beta \in \Pi\left(v^{*}\right)$.

\section{References}

[1] Aliprantis, C.D., Brown, and D. J., Burkinshaw, O.: Edgeworth equilibria, Econometrica 55 1109-1137 (1987)

[2] Aubin, J.P.: Mathematical Methods of Games and Economic Theory. North Holland, Amsterdam/New York/Oxford, 1979

[3] Bonnisseau, J.-M., V. Iehlé: Payoffs-dependent Balancedness and Cores, Cahiers de la MSE Série Bleue \# 2003.45, Université Paris-I (2003)

[4] Debreu, G., Scarf, H.: A limit theorem on the core of an economy. International Economic Review 4, 235-246 (1963)

[5] Edgeworth, F. Y.: Mathematical Psychics, Kegan Paul Publishers, London, (1881)

[6] Florenzano, M.: Edgeworth equilibria, fuzzy core and equilibria of a production economy without ordered preferences, Journal of Mathematical Analysis and Applications 153, 18-36 (1990)

[7] Predtetchinski, A., and P.J.J. Herings.: A Necessary and Sufficient Condition for the Non-emptiness of the Core of a Non-transferable Utility Game, Journal of Economic Theory 116, 84-92 (2004) 
[8] Predtetchinski, A.: The Fuzzy Core and the ( $\Pi, \beta)$-Balanced Core, Economic Theory 26, 717-724 (2005).

[9] Shapley, L., Vohra, R.: On Kakutani's fixed point theorem, the K-K-M theorem and the core of a balanced game, Economic Theory 1, 108-116, (1991)

[10] Vohra, R.: On Scarf's theorem on the non-emptiness of the core: A direct proof through Kakutani's fixed point theorem, Brown University Working Paper, (1987) 


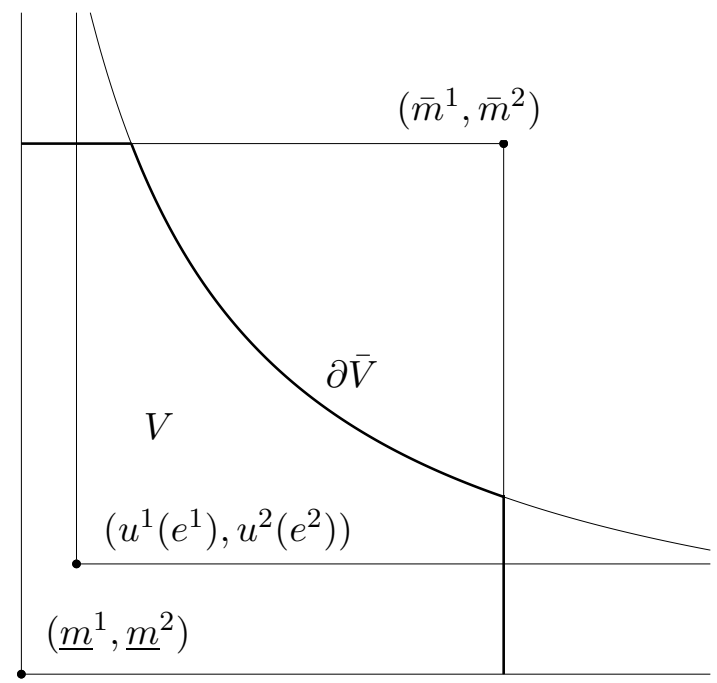

Figure 1: The boundary of the set set $\bar{V}$. 